\title{
RECENT DEVELOPMENTS IN PROJECTIVE DIFFERENTIAL GEOMETRY*
}

\author{
BY E. B. STOUFFER AND E. P. LANE
}

A. Invariants and Covariants, Canonical Forms, Canonical Expansions

1. Introduction. In a long series of papers appearing first in 1901 and continuing for more than twenty years, Wilczynski made a study of the projective differential properties of many geometrical figures, including plane and space curves, ruled and curved surfaces, and linear congruences. While Wilczynski was not the first to discover projective differential properties of figures, he was the first to proceed in a systematic manner in finding these properties. His general scheme in studying a given figure is to set up a system of one or more linear homogeneous differential equations such that the fundamental sets of solutions of the system determine the figure uniquely except for projective transformations. The independent and the dependent variables which appear in the differential equations and also in the parametric equations of the figure can be subjected to certain transformations which do not disturb the figure or change the form of the system of differential equations, but will in general change the coefficients of the differential equations. A function of the new coefficients and their derivatives and of the new dependent variables and their derivatives which is equal, except possibly for a factor, to the same function of the original coefficients and variables is called a covariant. A covariant which does not contain the dependent variables or their derivatives is called an invariant.

Wilczynski uses a complete and independent system of invariants and covariants as a foundation for each of his

${ }^{*}$ Two addresses presented at the request of the program committee at the Western meeting of the Society, April 6, 1928. Part A was given by Professor Stouffer, and Part B by Professor Lane. 
geometrical studies. His general method of calculating the invariants and covariants is by means of the Lie theory of continuous groups, a process which is frequently very laborious, although the final results are usually quite elegant. Since the method is purely analytical, there is no assurance that the invariants and covariants will appear in a form which will readily show their geometric significance.

Fubini and his school began about 1913 to study projective differential properties by means of differential forms. However, before many geometrical results can be obtained by this method it is necessary in each case to pass from the forms to a system of differential equations of the type used by Wilczynski. Fubini confines his attention almost wholly to systems which have been made canonical, that is, systems in which the coefficients have been specialized by means of the permissible transformations in such a way as to make the fundamental covariants take very simple forms.

One purpose of this paper is to derive several canonical forms for defining systems of differential equations and to show how each properly chosen canonical form leads in a very simple manner to a complete system of invariants and covariants of the Wilczynski type. A second purpose is to show how such canonical forms make possible direct derivation of canonical expansions for the equations of the figures.

In a paper ${ }^{(1)}$ published in 1915 Green showed how the invariants and covariants associated with a curved surface referred to general parametric curves could be obtained from those associated with the surface referred to the asymptotic curves as parametric curves. The principle which Green used in that particular problem is of remarkably wide application. We shall first apply it to the very simple case of a plane curve.

2. Curves. The differential equation associated with a plane curve $C$ is of the form ${ }^{(2)}$

$$
y^{\prime \prime \prime}+3 p_{1} y^{\prime \prime}+3 p_{2} y^{\prime}+p_{3} y=0,
$$

(1) Numerical references are made to the bibliography at the end. 
where differentiation is with respect to the independent variable $x$ and where $p_{i}$ are functions* of $x$. The permissible transformations in this case have the form

$$
\begin{aligned}
& \bar{x}=\phi(x), \\
& y=\lambda \bar{y},
\end{aligned}
$$

where $\phi$ and $\lambda$ are arbitrary functions of the independent variable.

The transformation (2) changes (1) into a new equation (1a) with coefficients $\bar{p}_{i}$ given by

(4) $\left\{\begin{array}{l}\bar{p}_{1}=\frac{1}{\phi^{\prime}}\left(p_{1}+\frac{\phi^{\prime \prime}}{\phi^{\prime}}\right), \\ \bar{p}_{2}=\frac{1}{\left(\phi^{\prime}\right)^{2}}\left(p_{2}+p_{1} \frac{\phi^{\prime \prime}}{\phi^{\prime}}+\frac{\phi^{\prime \prime \prime}}{3 \phi^{\prime}}\right), \quad \bar{p}_{3}=\frac{1}{\left(\phi^{\prime}\right)^{3}} p_{3}{ }^{3},\end{array}\right.$

and (3) in turn changes (1a) into another equation (1b) in $\bar{y}$ with coefficients $\bar{P}_{i}$ given by $\dagger$

$$
\left\{\begin{array}{l}
\bar{P}_{1}=\bar{p}_{1}+\frac{\bar{\lambda}^{\prime}}{\bar{\lambda}}, \\
\bar{P}_{2}=\bar{p}_{2}+2 \bar{p}_{1} \cdot \frac{\bar{\lambda}^{\prime}}{\bar{\lambda}}+\frac{\bar{\lambda}^{\prime \prime}}{\bar{\lambda}}, \\
\bar{P}_{3}=\bar{p}_{3}+3 \bar{p}_{2} \frac{\bar{\lambda}^{\prime}}{\bar{\lambda}}+3 \bar{p}_{1} \frac{\bar{\lambda}^{\prime \prime}}{\bar{\lambda}}+\frac{\bar{\lambda}^{\prime \prime \prime}}{\bar{\lambda}} .
\end{array}\right.
$$

It is easy to determine from (4) and (5) an invariant (2), that is, a function $\theta_{3}$ of $p_{i}$ which is related to the same function $\bar{\theta}_{3}$ of $\bar{P}_{i}$ by the equation $\bar{\theta}_{3}=\theta_{3} /\left(\phi^{\prime}\right)^{3}$.

If we choose $\phi^{\prime}$ to satisfy the equation $\ddagger\left(\phi^{\prime}\right)^{3}=\theta_{3}$ so that $\bar{\theta}_{3}=1$ and then choose $\bar{\lambda}$ to satisfy the equation $\bar{\lambda}^{\prime} / \bar{\lambda}=-\bar{p}_{1}$

*Here as everywhere else in this paper we shall assume that all functions which appear are capable of differentiation to as high an order as neccessary.

$\dagger$ In equations (5), $\bar{\lambda}$ is a function of $\bar{x}$ and differentiation is with respect to $\bar{x}$.

$\ddagger$ It is assumed that $C$ is not a conic, since in that case $\theta_{3}=0$. 
so that $\bar{P}_{1}=0$, we have a canonical form of the desired type for our differential equation. This is exactly the canonical form obtained by Sannia ${ }^{(3)}$ who introduced the differential form $a_{1} d x$ and then chose $a_{1}=\theta_{3}{ }^{1 / 3}$.

Since the conditions $\bar{\theta}_{3}=1, \bar{P}_{1}=0$ are maintained by the transformations (2) and (3) only if $\phi^{\prime \prime}=\lambda^{\prime}=0$, the coefficients $\bar{P}_{i}$ of our canonical form and their derivatives are each determined except for possible factors. A simple process of reasoning shows that they are the canonical forms of invariants whose general forms in terms of the coefficients of (1) can be obtained from (5) by direct substitutions from (4) with the now known values of $\phi^{\prime}$ and $\lambda^{\prime}$ inserted. The same reasoning shows that $\bar{y}$ and its derivatives are the canonical forms of covariants whose general forms follow by mere substitution. The completeness of the system of invariants and covariants thus obtained is evident and the determination of the particular invariants and covariants which are independent is not at all difficult.

The system of invariants and covariants thus obtained is, of course, equivalent to the system derived by Wilczynski and is, in fact, not greatly different from it. The great value of the method outlined above lies in its simplicity and in its complete avoidance of the complicated Lie theory.

A canonical form of the above type for the differential equation of a plane curve leads by a process involving only direct substitutions to an equation for the curve in the form of an expansion of one non-homogeneous coordinate in terms of the other. ${ }^{(4)}$ This method of obtaining a canonical expansion has the advantage that the vertices of the triangle of reference are exactly the points determined by the three covariants $\bar{y}, \bar{y}^{\prime}, \bar{y}^{\prime \prime}$ and are, as a consequence, easily characterized geometrically. Since a canonical expansion is the most natural and simple tool for obtaining results in this geometry, the ability to derive the expansion in a direct analytic manner is of great advantage.

The general scheme applied above to plane curve theory can be applied to space curves. ${ }^{(4)}$ However, it is desirable 
in this case to use the canonical expansion in order to determine the most satisfactory canonical form for the fundamental differential equations, even though this canonical form is actually used to determine the canonical expansion. We shall illustrate this principle in some detail by means of ruled surface theory. In the study of ruled surfaces it has been long and difficult work to determine the invariants and covariants( ${ }^{(2)}$ and a canonical expansion ${ }^{(5)}$ by previous methods.

3. Ruled Surfaces. Let us denote by $P$ the point on a ruled surface $R$ in the neighborhood of which we wish to study the surface. If we choose as one directrix curve $C_{y}$ the asymptotic curve through $P$ and for the other directrix curve $C_{z}$ any curve on the surface, it is well known ${ }^{(2)}$ that the fundamental system of differential equations has the general form

$$
\begin{aligned}
y^{\prime \prime}+2 p_{11} y^{\prime}+q_{12} z & =0, \\
z^{\prime \prime}+2 p_{21} y^{\prime}+2 p_{22} z^{\prime}+q_{21} y+q_{22} z & =0,
\end{aligned}
$$

where $p_{i j}$ and $q_{i j}$ are functions of the independent variable $x$. The permissible transformations are

$$
\bar{x}=\phi(x),
$$

for the independent variable and

$$
y=\alpha \bar{y}, \quad z=\gamma \bar{y}+\delta \bar{z}, \quad \alpha \delta \neq 0,
$$

for the dependent variables, where $\phi, \alpha, \gamma, \delta$ are arbitrary functions of the independent variable.

Let us now assume that (6) has been transformed by (7) and (8) into a canonical form with the dependent variables and their first derivatives uniquely determined except for possible factors. What the canonical form is we do not know as yet. However, it is easy to determine that the associated canonical expansion for the equation of the surface in the neighborhood of the point $P$ is

(9) $\zeta=\xi \eta+\frac{1}{3} \bar{Q}_{12} \xi^{3}+\frac{1}{12} \bar{V}_{12} \xi^{4}-\frac{1}{3}\left(\bar{U}_{11}-\bar{U}_{22}\right) \xi^{3} \eta+\cdots$, 
where $\bar{V}_{12}$ and $\bar{U}_{11}-\bar{U}_{22}$ are simple functions of the coefficients $\bar{P}_{i j}$ and $\bar{Q}_{i j}$ of the canonical form and their derivatives. Equation (9) makes evident the desirable conditions to be imposed in order to produce a canonical form and at the same time simplify the expansion. By means of (7) and (8) we can make*

$$
\bar{V}_{12}=\bar{U}_{11}-\bar{U}_{22}=\bar{P}_{11}=\bar{P}_{22}=0, \bar{Q}_{12}=1 .
$$

Since these conditions are maintained by (7) and (8) only if $\phi^{\prime \prime}=\alpha^{\prime}=\delta^{\prime}=\gamma=0$, we obtain for (6) a canonical form

$$
\left\{\begin{array}{l}
\bar{y}^{\prime \prime}+\quad \bar{Q}_{11} \bar{y}+\bar{z}=0, \\
\bar{z}^{\prime \prime}+2 \bar{P}_{21} \bar{y}^{\prime}+\bar{Q}_{21} \bar{y}+\bar{Q}_{11} \bar{z}=0
\end{array}\right.
$$

in which the coefficients and their derivatives are invariants and the dependent variables and their derivatives are covariants, all in their canonical forms. The general form of this complete system of invariants and covariants in terms of the coefficients and variables of (6) can be obtained by direct substitutions just as the case of plane curves.

The expansion (9) is thus reduced to the simple form $\zeta=\xi \eta+\xi^{3} / 3+$ terms of at least the fifth degree. The vertices of the tetrahedron of reference are given at once by the four simplest covariants. Their geometrical significance is easily determined. In fact, one vertex is the point $P$, a second is the point $\bar{P}_{z}$, the harmonic conjugate of $P$ with respect to the flecnode points on the generator through $P$, a third is the point $\bar{P}_{\rho}$ on the tangent to $C_{y}$ at $P$ which is the pole of the generator with respect to the osculating conic of $C_{y}$ at $P$, and the fourth is the intersection of the asymptotic tangent at $\bar{P}_{z}$ and the line of the osculating hyperboloid which passes through $\bar{P}_{\rho}$.

The geometrical significance of the vanishing of the invariants, which in their canonical forms are the coefficients of (10), can be read directly from the differential equation.

*These results are not valid if $P$ is a point on a flecnode curve. 
In fact, if $\bar{P}_{21}=0, \bar{C}_{z}$ is an asymptotic curve, if $\bar{Q}_{21}=0$ the flecnode curves coincide and if $\bar{Q}_{11}=0$ the tangent at $\bar{P}_{\rho}$ to $\bar{C}_{\rho}$ passes through $\bar{P}_{z}$.

$\check{C} \mathrm{ech}^{(6)}$ has applied Fubini's methods to ruled surfaces by starting with a specialized case of the differential forms for curved surfaces. He derives from these forms a pair of second order differential equations which he makes canonical by putting a certain invariant $\theta_{4}$ of Wilczynski equal to unity.

4. Curved Surfaces. In applying the above methods to curved surfaces we start with the fundamental equations ${ }^{(7)}$

$$
\begin{aligned}
& y_{u u}+2 a y_{u}+2 b y_{v}+c y=0, \\
& y_{v v}+2 a^{\prime} y_{u}+2 b^{\prime} y_{v}+c^{\prime} y=0,
\end{aligned}
$$

with the asymptotic curves parametric. The permissible transformations are

$$
\bar{u}=\phi(u), \quad \bar{v}=\psi(v),
$$

for the independent variables, and

$$
y=\lambda(u, v) \bar{y},
$$

for the dependent variable.

Let us assume that (11) has been transformed by (12) and (13) into a system with coefficients $\bar{A}, \bar{B}, \bar{C}, \bar{A}^{\prime}, \bar{B}^{\prime}, \bar{C}^{\prime}$, whose dependent variable $\bar{y}$ and its derivatives $\bar{y}_{\bar{u}}, \bar{y}_{\bar{v}}, \bar{y}_{\bar{u}} \bar{v}$ are all completely determined except for possible factors. It is easily found that an associated canonical expansion for the equation of the surface $S$ in the neighborhood of a point $\bar{P}_{y}$ given by $\bar{u}=\bar{u}_{0}, \bar{v}=\bar{v}_{0}$, is

$$
\left\{\begin{array}{l}
\zeta=\xi \eta+\frac{2}{3} \bar{B} \xi^{3}+\frac{2}{3} \bar{A}^{\prime} \eta^{3}+\frac{2}{3}\left(\bar{B}_{\bar{v}}-2 \bar{B} \bar{B}^{\prime}\right) \xi^{3} \eta \\
+\frac{2}{3}\left(\bar{A}^{\prime} \frac{1}{u}-2 \overline{A A}^{\prime}\right) \xi \eta^{3}+\frac{1}{6}\left(\bar{B}_{\bar{u}}+4 \bar{A} \bar{B}\right) \xi^{4} \\
+\frac{1}{6}\left(\bar{A}^{\prime} \frac{1}{v}+4 \bar{A}^{\prime} \bar{B}^{\prime}\right) \eta^{4}+\frac{1}{2}\left(\bar{A}_{\bar{v}}+\bar{B}_{\bar{u}}\right) \xi^{2} \eta^{2}+\cdots
\end{array}\right.
$$

The fact that $\phi(u)$ and $\psi(v)$ in the transformation (12) are each functions of only a single variable limits the conditions which may be imposed by this transformation. However, we can, for instance, impose by (12) and (13) the conditions 


$$
\begin{aligned}
\bar{A}^{\prime}\left(\bar{u}, \bar{v}_{0}\right) & =\bar{B}_{\bar{v}}\left(\bar{u}_{0}, \bar{v}\right)=0, \\
\bar{A} & =\bar{B}^{\prime}=0,
\end{aligned}
$$

conditions which are maintained by (12) and (13) only if $\phi_{u u}=\psi_{v v}=\lambda_{u}=\lambda_{v}=0$. Since the coefficients in (14) are functions of $\bar{u}_{0}$ and $\bar{v}_{0}$ we are thus led to a canonical expansion for the equations of the surface. However, we do not obtain in place of (11) a canonical form in the true sense. Nevertheless, by the same kind of simple substitutions as above, the coefficients and variables of the reduced form and their derivatives produce a complete system of invariants and covariants in terms of the coefficients and variables of (11). The expansion (14) becomes under the conditions (15) and (16) essentially that obtained by Wilczynski( ${ }^{(8)}$ and also by Green $^{(9)}$, with the vertices of the tetrahedron of reference determined by the simplest covariants and located at the intersection of Wilczynski's directrices of the first and second kind with his canonical quadric ${ }^{(8)}$.

In place of (15) we may impose the more general conditions

$$
l \frac{\bar{A}^{\prime} \bar{u}\left(\bar{u}, \bar{v}_{0}\right)}{\bar{A}^{\prime}\left(\bar{u}, \bar{v}_{0}\right)}+m \frac{\bar{B} \bar{u}\left(\bar{u}, \bar{v}_{0}\right)}{\bar{B}\left(\bar{u}, \bar{v}_{0}\right)}=l \frac{\bar{B}_{\bar{v}}\left(\bar{u}_{0} \bar{v}\right)}{\bar{B}\left(\bar{u}_{0}, \bar{v}\right)}+m \frac{\bar{A}_{\hat{v}}^{\prime}\left(\bar{u}_{0}, \bar{v}\right)}{\bar{A}^{\prime}\left(\bar{u}_{0}, \bar{v}\right)}=0
$$

where $l$ and $m$ are constants. In each case we obtain a canonical expansion with two points $\bar{P}_{z}$ and $\bar{P}_{\rho}$, one on each asymptotic tangent through $P_{y}$, as two vertices of the tetrahedron of reference. The fourth vertex $\bar{P}_{\sigma}$ is the intersection of the canonical quadric of Wilczynski with the polar reciprocal of the line $\bar{P}_{z} \bar{P}_{\rho}$ with respect to the quadric of Lie. For all values of $l$ and $m$ the lines joining corresponding points $\bar{P}_{z}$ and $\bar{P}_{\rho}$ pass through a point, the canonical point, and the corresponding lines joining $P_{y}$ and $\bar{P}_{\sigma}$ all lie in a plane, the canonical plane.

If $m=0$ we have the case mentioned above. If $l=0$ the vertices are on the canonical edges of Green ${ }^{(9)}$. If $l / m=1$ the line joining $P_{y}$ and $\bar{P}_{\sigma}$ is the pseudo-normal of Green ${ }^{(9)}$, the projective normal of Fubini(10). Other values of the 
ratio $l / m$ give other covariant lines. In each case the coefficients and variables of the reduced form produce a corresponding system of invariants and covariants which can be expressed in terms of the coefficients and variables of (11) by direct substitutions.

If we apply the transformations (13) alone, we find that it is possible to impose the conditions ${ }^{(11)}$

$$
2 \bar{A}+\left(\log \bar{A}^{\prime} \bar{B}\right)_{u}=2 \bar{B}^{\prime}+\left(\log \overline{A^{\prime}} \bar{B}\right)_{v}=0 .
$$

These conditions are not disturbed by the general transformation (12). If we write $\theta=(1 / 2) \log \bar{A}^{\prime} \bar{B}$, equations (11) become under the conditions (18)

$$
\begin{aligned}
\bar{y}_{u u}-2 \theta_{u} \bar{y}_{u}+2 \bar{B} \bar{y}_{v}+\bar{C} \bar{y} & =0, \\
\bar{y}_{v v}+2 \bar{A}^{\prime} \bar{y}_{u}-2 \theta_{v} \bar{y}_{v}+\bar{C}^{\prime} \bar{y} & =0,
\end{aligned}
$$

which is the canonical form of Fubini.

If $\bar{A}$ and $\bar{B}^{\prime}$ are replaced by $-\theta_{u}$ and $-\theta_{v}$, respectively, in accordance with (18), the expansion (14) becomes essentially the canonical expansion of Fubini ${ }^{(12)}$. The vertices of the tetrahedron of reference for this expansion are the same as for the case $l / m=1$ above, except that the fourth vertex is the intersection of the projective normal with Fubini's canonical quadric ${ }^{(20)}$. Fubini ${ }^{(13)}$ has derived the same expansion without the use of a fundamental system of differential equations. He simply assumes an expansion of one non-homogeneous coordinate in terms of the other two and then chooses his tetrahedron of reference properly.

Recently Lane ${ }^{(14)}$ has obtained all the above mentioned canonical expansions for the equation of a curved surface, starting from the equivalent of equations (18). The same author ${ }^{(39)}$ has also derived a significant canonical form for the equations of a curved surface referred to a conjugate net as the parametric net.

The methods outlined above for obtaining complete systems of invariants and covariants and canonical expansions apply equally well in many other cases. However, their consideration must be left to other occasions. 


\section{B. Geometry of Surfaces}

1. Surfaces in Ordinary Space. The foundations for a theory of the projective differential geometry of an analytic non-ruled surface in ordinary space were laid by Wilczynski in a series of five memoirs which were published in the Transactions of this Society from 1907 to 1909. As the theory grew and expanded, Green made notable contributions to it, especially in a memoir that appeared in the Transactions after his death in 1919. The work of Fubini on this subject dates from about 1913, and that of Bompiani from about 1923.

A considerable portion of the projective differential geometry of a surface as elaborated in the last two decades, together with some of the more or less incidental results found by various geometers before 1907 can be organized about three unifying ideas, namely, quadrics containing the asymptotic tangents, the canonical pencils of lines, and union curves with their generalizations. After establishing an analytic basis for our discussion we shall consider each of these topics in turn.

Let the projective homogeneous coordinates $x^{(1)}, \cdots, x^{(4)}$ of a point $P$ on a surface $S$ be functions of two independent variables $u, v$. If the asymptotic net is parametric and if the proportionality factor of the coordinates is suitably chosen, then the functions $x$ are solutions of a completely integrable system of differential equations of the form

$$
\begin{array}{r}
x_{u u}=p x+\theta_{u} x_{u}+\beta x_{v}, \quad x_{v v}=q x+\gamma x_{u}+\theta_{v} x_{v}, \\
(\theta=\log \beta \gamma) .
\end{array}
$$

The coordinates of a point $N$ on $S$ near $P$ can be represented by Taylor's formula as power series in the increments $\Delta u$, $\Delta v$ corresponding to displacement from $P$ to $N$. Then by means of (1) and the equations obtained therefrom by differentiation it is possible to express each of these series uniquely in the form $x_{1} x+x_{2} x_{u}+x_{3} x_{v}+x_{4} x_{u v}$, where $x_{1}, \cdots$, $x_{4}$ are the following series which represent the local coordi- 
nates of $N$ referred to the covariant tetrahedron $x, x_{u}, x_{v}$, $x_{u v}$, with suitably chosen unit point:

$$
\left\{\begin{array}{l}
x_{1}=1+\left(p \Delta u^{2}+q \Delta v^{2}\right) / 2+\cdots \\
x_{2}=\Delta u+\left(\theta_{u} \Delta u^{2}+\gamma \Delta v^{2}\right) / 2+\cdots \\
x_{3}=\Delta v+\left(\beta \Delta u^{2}+\theta_{v} \Delta v^{2}\right) / 2+\cdots \\
x_{4}=\Delta u \Delta v+\left(\beta \Delta u^{3}+3 \theta_{u} \Delta u^{2} \Delta v+3 \theta_{v} \Delta u \Delta v^{2}+\gamma \Delta v^{3}\right) / 6+\cdots
\end{array}\right.
$$

The equations of the $u$-tangent are $x_{3}=x_{4}=0$, and those of the $v$-tangent are $x_{2}=x_{4}=0$.

2. Quadrics containing the Asymptotic Tangents. The equation of any non-singular quadric surface $\phi$ containing the asymptotic tangents of $S$ at $P$ can be written ${ }^{(15)}$ in the form

$$
x_{2} x_{3}+x_{4}\left(K_{1} x_{1}+K_{2} x_{2}+K_{3} x_{3}+K_{4} x_{4}\right)=0 \quad\left(K_{1} \neq 0\right) .
$$

If $K_{1}=-1$, then $\phi$ has contact of the second order with $S$ at $P$ and cuts $S$ in a curve with a triple point at $P$. If the triple point tangents coincide, they coincide in one of the three directions of Darboux ${ }^{(16)}$ for which

$$
\beta d u^{3}+\gamma d v^{3}=0 .
$$

If $K_{1}=-1$ and $K_{2}=K_{3}=0$, then $\phi$ is a quadric of Darboux, which has contact of the second order and has the tangents of Darboux for triple point tangents.

Among the quadrics of Darboux there are four that deserve mention. If $K_{4}=-\left(\beta \gamma+\theta_{u v}\right) / 2$, then $\phi$ is the quadric of $L_{i e^{(17)}}$, called by Wilczynski the osculating quadric, which is the limit of the quadric determined by three asymptotic tangents of one family constructed at points of a fixed curve of the other family as these points approach coincidence along the fixed asymptotic. If $K_{4}=-\theta_{u v} / 2$, then $\phi$ is the canonical quadric of Wilczynski(8). Bompiani has recently ${ }^{(18)}$ rediscovered this quadric, apparently without recognizing it, by means of the following considerations. If a curve $C$ on $S$ has an inflexion at $P$ then $C$ is tangent to an asymptotic at $P$. The limit of the quadric determined 
by three asymptotic tangents of the other family constructed at points of $C$, as these points approach $P$ along $C$, is the canonical quadric of Wilczynski. If in this definition the curve $C$, instead of having an inflexion, has a stationary osculating plane at $P$, the resulting quadric is the quadric of Fubini(19) for which $K_{4}=-\left(2 \beta \gamma+3 \theta_{u v}\right) / 6$. Finally, if $K_{4}=0$, then $\phi$ is the canonical quadric of Fubini ${ }^{(20)}$, which is the quadric of Darboux that passes through the covariant point $(0,0,0,1)$.

We shall refer briefly to only two more species of quadrics of the general type (3). At a point $P$ of curve $C$ on $S$ there are two of Bompiani's asymptotic osculating quadrics ${ }^{(21)}$; each of these is the limit of the quadric determined by three asymptotic tangents of one family constructed at points of $C$, as these points independently approach $P$ along $C$. And the quadric of Moutard ${ }^{(22)}$ of $S$ at $P$ in the direction of a tangent $t$ is the locus of the osculating conics at $P$ of the curves of intersection of $S$ and the planes of the pencil with $t$ as axis.

3. The Canonical Pencils. The canonical line pencils of $S$ at $P$ may be defined analytically as follows. The line $l_{1}$ joining $P$ to the point $(0, K \psi, K \phi, 1)$, where

$$
\phi=\frac{\partial}{\partial u} \log \beta \gamma^{2}, \quad \psi=\frac{\partial}{\partial v} \log \beta^{2} \gamma, \quad K=\text { const } .
$$

is a canonical line of the first kind. When $K$ varies, the locus of $l_{1}$ is the first canonical pencil, with center at $P$ and lying in the canonical plane $\phi x_{2}-\psi x_{3}=0$. The polar line $l_{2}$ of $l_{1}$ with respect to the quadric of Lie is a canonical line of the second kind and joins the points $(K \phi, 1,0,0),(K \psi, 0,1,0)$. When $K$ varies, the locus of $l_{2}$ is the second canonical pencil with center at the canonical point $(0, \psi,-\phi, 0)$ and lying in the tangent plane $x_{4}=0$.

Every canonical line is covariant to the surface, but negative rational values of $K$ give most of the lines that have appeared naturally in geometric investigations. For instance, 
if $K=-1 / 2$, then $l_{1}, l_{2}$ are the directrices ${ }^{(8)}$ of Wilczynski. These have several characteristic properties, but were discovered by Wilczynski as the directrices of the linear congruence of intersection of the osculating linear complexes of the two asymptotic curves at $P$. If $K=-1 / 4$, then $l_{1}, l_{2}$ are the canonical edges ${ }^{(9)}$ of Green. Perhaps the most recently discovered characteristic property of these lines is due to B. Segre who shows ${ }^{(23)}$ that all non-composite cubic surfaces having contact of the fourth order with $S$ at $P$ cut the tangent plane in the same cubic curve which has a double point at $P$ and has three inflexions lying on the second canonical edge of Green. If $K=-1 / 3$, then $l_{1}$ is the axis of Cech, called ${ }^{(24)}$ by him the line of Segre, because the osculating planes of the three curves of Segre $\beta d u^{3}-\gamma d v^{3}=0$ at $P$ intersect in this line. If $K=0$, then $l_{1}$ is the projective normal ${ }^{(10)}$ of Fubini, which was discovered independently by Green and called(9) by him the pseudo-normal. Other canonical lines of interest are the principal lines ${ }^{(25)}$ of $\mathrm{Fu}$ bini, for which $K=-1 / 6, K=-1 / 12$; the lines ${ }^{(15)}$ for which $K=-5 / 12, K=-3 / 4$; and ${ }^{(26)}$ the lines ${ }^{(27)}$ for which $K=-3 / 8$.

4. Union Curves. Let us consider a congruence $\Gamma$ of lines one of which, $l$, passes through each point $P$ of $S$ but does not lie in the tangent plane at $P$. A union curve of $\Gamma$ is defined $^{(28)}$ by Miss Sperry to be a curve on $S$ such that its osculating plane at each of its points contains the line of $\Gamma$ through the point. The differential equation of the union curves has the form

$$
v^{\prime \prime}=A+B v^{\prime}+C v^{\prime 2}+D v^{\prime 3} \quad\left(v^{\prime}=d v / d u\right),
$$

and the osculating planes at $P$ of all the union curves of $\Gamma$ through $P$ form a pencil with the line $l$ through $P$ as axis. For this reason Bompiani ${ }^{(29)}$ calls the union curves of a congruence an axial system. In metric geometry the union curves of the congruence of normals are the geodesics.

The most general equation of the form (6) defines on $S$ a system of curves such that the osculating planes at a point 
$P$ of all the curves of the system that pass through $P$ envelope a cone of the third class which touches the tangent plane of $S$ along the asymptotic tangents at $P$, and which has three cusp-planes that intersect in a line through $P$, called the cusp-axis of $P$ with respect to the given system of curves. We shall mention two examples of cusp-axes. The extremals of the integral

$$
\int\left(\beta \gamma v^{\prime}\right)^{1 / 2} d u
$$

are given by Euler's equation,

$$
v^{\prime \prime}=\theta_{u} v^{\prime} / \theta-\theta_{v} v^{\prime 2} / \theta, \quad(\theta=\log \beta \gamma),
$$

and the corresponding cusp-axis is the projective normal. The curves of the Segre-Darboux pencil

$$
v^{\prime \prime}=\lambda_{u} v^{\prime} / \lambda+\lambda_{v} v^{\prime 2} / \lambda, \quad\left(\lambda=(\beta / \gamma)^{1 / 3}\right) .
$$

have for cusp-axis the axis of Cech.

Bompiani defines ${ }^{(30)}$ a planar system of curves as follows. Let us consider a congruence $\Gamma$ of quadrics one of which is associated with each point $P$ of $S$ and contains the asymptotic tangents through $P$ but does not have contact of order as high as the second with $S$ at $P$. Then a planar curve of $\Gamma$ is a curve on $S$ such that at each of its points its asymptotic osculating quadric of one family (which contains the asymptotic tangents through the point) intersects the associated quadric of $\Gamma$ in a residual pair of straight lines, instead of in a residual non-singular conic as would ordinarily be the case. The differential equation of a planar system is of the form (6). A given congruence $\Gamma$ determines two planar systems of curves because a curve has two families of asymptotic osculating quadrics. But if a planar system is defined by one family of asymptotic osculating quadrics and a congruence $\Gamma$, then a second congruence $\Gamma$ can be determined so that it and the other family of asymptotic osculating quadrics define the same planar system. The reason for 
the name planar system will appear when we discuss Bompiani's extension of this theory to hyperspace.

5. Method of the Italian School. Italian geometers of the school of Fubini define a configuration, except for a projective transformation, by means of a system of differential forms, and employ the absolute calculus of Ricci; whereas American geometers of the school of Wilczynski define a configuration projectively by means of a system of differential equations and make use of the Lie theory of continuous groups. Fubini's method ${ }^{(31)}$ has been used by Sannia in studying plane and space curves; by Čech in his theory of ruled surfaces; and by Fubini in his investigations of rectilinear congruences and complexes. But the method finds perhaps its best exemplification in Fubini's theory of surfaces in ordinary space. We shall sketch this theory very briefly.

Let us consider a fundamental binary quadratic differential form

(9) $G=a_{i j} d u_{i} d u_{j}, \quad\left(a_{i j}=a_{j i} ; A=\left|a_{i j}\right| \neq 0 ; i, j=1,2\right)$,

which will be completely specified later, and let us also consider a surface $S$ whose parametric equations in projective homogeneous point coordinates are given by the equations $x^{(k)}=x^{(k)}(u, v)(k=1, \cdots, 4)$. Let us define two differential forms $F_{2}, \Phi_{3}$ by the equations

(10) $F_{2}=\left(x, x_{1}, x_{2}, d^{2} x\right)|A|^{-1 / 2}, \Phi_{3}=\left(x, x_{1}, x_{2}, d^{3} x\right)|A|^{-1 / 2}$,

wherein numerical subscripts of $x$ indicate covariant differentiation with respect to $G$, and a determinant is indicated by writing a typical row within parentheses. The forms $F_{2}$, $\Phi_{3}$ are of the first and second orders respectively, and are both absolutely invariant under transformation of curvilinear coordinates $u, v$ on $S$. But under transformation of fundamental form $G$ and of proportionality factor $\rho$ of the homogeneous coordinates, the forms $F_{2}, \Phi_{3}$ behave quite differently. 
Let us define a differential form $F_{3}$ by the equation

$$
F_{3}=2 \Phi_{3}-3 d F_{2}+\frac{3}{4} F_{2} d \log \frac{\Delta}{A},
$$

where $\Delta$ is the discriminant of $F_{2}$. Both forms $F_{2}, F_{3}$ are of the first order of degrees indicated by the subscripts, are absolutely invariant under transformation of curvilinear coordinates, and are cogredient under transformation of $G$ and $\rho$. In fact both forms are relative invariants under the latter transformation, since we have

$$
\left|A^{\prime}\right|^{1 / 2} F_{i}^{\prime}=\rho^{4}|A|^{1 / 2} F_{i}, \quad(i=2,3),
$$

accents indicating the transformed quantities.

Let us define two forms $\phi_{2}, \phi_{3}$ by the formula

$$
\phi_{i}=\rho^{4}|A|^{1 / 2}\left|A^{\prime}\right|^{-1 / 2} F_{i}, \quad(i=2,3),
$$

wherein $\rho$ is chosen so that the ratio of the discriminant of $\phi_{3}$ to the cube of the discriminant of $\phi_{2}$ is constant. With this choice of $\rho$ the coordinates are Fubini's normal coordinates. Next let us choose the fundamental form $G$ to be $\phi_{2}$. Then the hessian of $\phi_{3}$ is proportional to $\phi_{2}$, so that $\phi_{3}$ is apolar to $\phi_{2}$. If the asymptotic net on $S$ is taken for parametric net, we can write

$$
\phi_{2}=2 \beta \gamma d u d v, \quad \phi_{3}=2 \beta \gamma\left(\beta d u^{3}+\gamma d v^{3}\right) .
$$

If we attempt to set up the differential equations to obtain a surface which has the forms $\phi_{2}, \phi_{3}$ arbitrarily assigned, we find that $\phi_{2}, \phi_{3}$ are not sufficient to define a surface to within a projective transformation. But if we adjoin to $\phi_{2}, \phi_{3}$ the form $\psi_{2}$ defined by

$$
\psi_{2}=p d u^{2}+q d v^{2},
$$

then the forms $\phi_{2}, \phi_{3}, \psi_{2}$ do define a surface, except for a projective transformation, and the differential equations for obtaining such a surface are precisely equations (1). At this point the Italian and American theories merge.

The form $\phi_{2}$ vanishes for the asymptotic curves, and $\phi_{3}$ 
for the curves of Darboux, while $\psi_{2}$ vanishes for the curves corresponding to the developables of the congruence of lines which are polars of the projective normals with respect to the quadrics of Lie.

6. Extensions to Hyperspace. Extensive investigations in the projective differential geometry of hyperspace have been made by Segre ${ }^{(32)}$, Bompiani, Fubini, Terracini, Tzitzéica ${ }^{(33)}$ and others. We shall confine our attention to only two of the many topics that might be considered, first discussing Bompiani's pluri-axial systems, and then comparing the Italian and American methods as applied in hyperspace.

7. Pluri-Axial Systems. In a linear space $S_{n}$ of $n$ dimensions let us consider a surface $S$ which has on it a conjugate net, so that at every point of $S$ the osculating planes of all the curves on $S$ through the point are contained in an $S_{4}$ instead of in the usual $S_{5}$. Let a congruence $\Gamma$ of planes be given, one of which passes through each point $P$ of $S$ and lies in the tangent $S_{4}$ without intersecting, except at $P$, the tangent plane of $S$ at $P$. Then a curve on $S$ is a planar curve $^{(34)}$ of $\Gamma$ in case its osculating plane at each of its points intersects in a straight line the plane of $\Gamma$ through the point. The differential equation of a planar system of curves has the form (6). It is well known that point geometry on a hyperquadric in $S_{5}$ is isomorphic with line geometry in ordinary ruled space $R_{3}$, the correspondance being established by interpreting the six Plückerian coordinates of a line in $R_{3}$ as the coordinates of a point in $S_{5}$. If the surface $S$ under consideration here is the surface in $S_{5}$ that corresponds to one congruence of asymptotic tangents of a surface in $R_{3}$, then the planar system of curves defined here corresponds to the plar ar system previously defined.

On a zeneral surface $S$ in $S_{n}$ a system of curves analogous to a planar system can be defined ${ }^{(35)}$ by considering a congruence of spaces $S_{3}$, instead of planes, one of which passes through each point $P$ of $S$ and lies in the tangent $S_{5}$ at $P$. More generally, let us consider at each point $P$ of $S$ 
the $S(k)$ which is the ambient space of the osculating $S_{k}$ at $P$ of every curve on $S$ through $P$. Then let a congruence $\Gamma$ of linear spaces $S_{h}$ be given, one of which passes through each point $P$ of $S$ and lies in the $S(k)$ at $P$. Then a curve on $S$ is a pluri-axial curve of $\Gamma$ in case its osculating $S_{k}$ at each of its points intersects the $S_{h}$ of $\Gamma$ through the point in a linear space of dimensions greater than would ordinarily be the case.

Still further generalizations can be obtained ${ }^{(36)}$ by replacing the surface $S$ by a variety $V_{k}$ of $k$ dimensions and associating with each point of $V_{k}$ a linear space $S_{h}$. Or instead of linear spaces $S_{h}$, cones may be used. And instead of a pluri-axial system of curves, it would seem possible to define a pluri-axial system of varieties $V_{j}$ on $V_{k}(1<j<k)$.

8. Comparison of Italian and American Methods. The analytic and synthetic methods used by Italian geometers in studying the projective differential geometry of hyperspace possess great power and elegance. Nevertheless there does not yet exist a general theory of a $V_{k}$ in $S_{n}$ comparable with the Italian and American theories of a surface in ordinary space. The Italian method of differential forms has failed for a $V_{k}$ in $S_{n}(1<k<n-1, n>4)$, either because of the lack of a covariant quadratic differential form, or because of the lack of an absolute calculus for an $n$-ary $p$-adic differential form. The American method of differential equations is theoretically available, but the amount of labor involved in making the necessary calculations seems at present to be practically prohibitive in so far as establishing a general theory is concerned. In special cases, however, the American method is applicable. For instance, Miss Beenken has recently ${ }^{(37)}$ applied this method in a study of surfaces in $S_{5}$. And there is hope that it may be possible to modify the American method so as to avoid much of the labor of the calculations ordinarily involved by reducing all of the differential equations of a defining system to equations of the first order ${ }^{(38)}$. 


\section{BIBLIOGRAPHY}

${ }^{1}$ Green, On the theory of curved surfaces, and canonical systems in projective differential geometry, Transactions of this Society, vol. 16 (1915), p. 1. This journal will be cited as Transactions.

${ }^{2}$ Wilczynski, Projective Differential Geometry of Curves and Ruled Surfacts, Leipzig, Teubner, 1906.

${ }^{3}$ Sannia, Nuova trattazione della geometria proiettivo-differenziale delle curve piane, Rendiconti della R. Accademia dei Lincei, (5), vol. 31 (1922), $1^{\circ}$ sem., p. 450 and p. $503 ; 2^{\circ}$ sem., p. 17 and p. 432 . This journal will be cited as Lincei.

${ }^{4}$ Stouffer, Some canonical forms and associated canonical expansions in projective differential geometry, this Bulletin, vol. 34 (1928), pp. 290-302.

${ }^{5}$ Wilczynski, Third Memoir, Transactions, vol. 9 (1908), p. 293.

${ }^{6}$ Fubini e Čech, Geometria Proiettiva Differenziale, Bologna, Zanichelli, 1926, chap. 4. This treatise will be cited as Fubini and Cech.

${ }^{7}$ Wilczynski, First Memoir, Transactions, vol. 8 (1907), p. 233.

${ }^{8}$ Wilczynski, Second Memoir, Transactions, vol. 9 (1908), p. 79.

${ }^{9}$ Green, Memoir on the general theory of surfaces and rectilinear congruences, Transactions, vol. 20 (1919), p. 79.

${ }^{10}$ Fubini, Fondamenti della geometria proiettivo-differenziale di una superficie, Atti della R. Accademia delle Scienze di Torino, vol. 53 (1918), p. 1032.

${ }^{11}$ Lane, Wilczynski's and Fubini's canonical systems of differential equations, this Bulletin, vol. 32 (1926), p. 365.

${ }^{12}$ Fubini, Invarianti proiettivo-differenziali delle curve tracciate su una superficie, e definizione proiettivo-differenziale di una superficie, Annali di Matematica, (3), vol. 25 (1916), p. 229.

${ }^{13}$ Fubini, Nuova trattazione elementare dei fondamenti della geometria proiettivo-differenziale di una superficie, Rendiconti del R. Istituto Lombardo di Scienze e Lettere, vol. 59 (1926), p. 69.

${ }^{14}$ Lane, Power series expansions in the neighborhood of a point on a surface, Proceedings of the National Academy of Sciences, vol. 13 (1927), p. 808.

${ }^{15}$ Lane, Quadriche aventi per generatrici le tangenti asintotiche in un punto di una superficie, Lincei, (6), vol. 5 (1927), p. 100.

${ }^{16}$ Darboux, Sur le contact des courbes et des surfaces, Bulletin des Sciences Mathématiques, (2), vol. 4 (1880), p. 348.

${ }^{17}$ Lie, Gesammelte Abhandlungen, vol. 3, 1922, p. 718.

${ }^{18}$ Bompiani, Fascio di quadriche di Darboux e normale proiettiva in un punto di una superficie, Lincei, (6), vol. 6 (1927), p. 187.

${ }^{19}$ Fubini, Una nuova generazione delle quadriche di Darboux, Lincei, (6), vol. 7 (1928), p. 14.

${ }^{20} \mathrm{~F}$ ubini and Čech, p. 129.

${ }^{21}$ Lane, The asymptotic osculating quadrics of a curve on a surface, this Bulletin, vol. 33 (1927), p. 195.

${ }^{22}$ Moutard, Comptes Rendus, vol. 91 (1880), p. 1055.

${ }^{23}$ B. Segre, Comptes Rendus, vol. 184 (1927), p. 729. 
24 Cech, L'intorno di un punto d'una superficie considerato dal punto di vista proiettivo, Annali di Matematica, (3), vol. 31 (1922), p. 191.

${ }^{25}$ Fubini, Alcune risultati di geometria proiettivo-differenziale, Lincei, (5), vol. 32 (1923), p. 273 and p. 321.

${ }^{26}$ Bompiani, Rappresentazione geodetico-proiettiva fra due superficie, Annali di Matematica, (4), vol. 3 (1925), p. 171.

${ }^{27}$ Lane, Le direttrici di Sullivan, Bollettino della Unione Matematica Italiana, vol. 5 (1926), p. 214.

${ }^{28}$ Sperry, Properties of a certain projectively defined two-parameter family of curves on a general surface, American Journal of Mathematics, vol. 40 (1918), p. 213.

${ }^{29}$ Bompiani, Proprietd generali della rappresentazione puntuale fra due superficie, Annali di Matematica, (4), vol. 1 (1923), p. 259.

${ }^{30}$ Bompiani, Ancora sulla geometria delle superficie considerate nello spazio rigato, Lincei, (6), vol. 4 (1926), p. 262.

${ }^{31}$ Fubini and Čech, vol. 1, chaps. 1, 2, 4, and vol. 2, chaps. 11, 12.

${ }^{32}$ Fubini and Čech, vol. 2, p. 729.

${ }^{33}$ Tzitzéica, Géométrie Différentielle Projective des Réseaux, Académie Roumaine, Etudes et Recherches, Bucarest, 1923.

${ }^{34}$ Bompiani, Sulla corrispondenza puntuale fra due superficie a punti planari, Bollettino della Unione Matematica Italiana, vol. 4 (1925), p. 195.

${ }^{36}$ Bompiani, Alcune idee gentrali per lo studio differenziale delle varietd, Lincei, (6), vol. 5 (1927), p. 383.

${ }^{36} \mathrm{E}$. Bortolotti, Sistemi assiali e connessioni nelle $V_{m}$, Lincei, (6), vol. 5 (1927), p. 390.

${ }^{37}$ Beenken, Surfaces in space of five dimensions, Doctoral dissertation, Chicago, 1928.

${ }^{38}$ Lane, Projective differential geometry of systems of linear homogeneous differential equations of the first order.

${ }^{39}$ Lane, Contributions to the theory of conjugate nets, American Journal of Mathematics, vol. 49 (1927), p. 565.

The University of Kansas,

The University of Chicago. 